\title{
The role of MRP1 in the multidrug resistance of colorectal cancer
}

\author{
DONGXING CAO, SHAOLAN QIN, YIFEI MU and MING ZHONG
}

\begin{abstract}
Department of Gastrointestinal Surgery, Renji Hospital Affiliated to Shanghai Jiao Tong University School of Medicine, Shanghai 200127, P.R. China
\end{abstract}

Received October 2, 2015; Accepted November 25, 2016

DOI: $10.3892 / \mathrm{ol} .2017 .5741$

\begin{abstract}
The role of multidrug resistance associated protein 1 (MRP1) in the multidrug resistance (MDR) of colorectal cancer (CRC) remains unclear. The present study aimed to investigate the effect of MRP1 in MDR CRC and its therapeutic potential for the treatment of patients with this disease. The human MDR CRC cell lines HCT-8 and Colo205 were established through stable exposure to 5-florouracil (5-FU) over a 5-month period. MRP1 was knocked-down in MDR CRC cells through the transfection of short hairpin RNA targeting MRP1 (shMRP1). Western blotting was performed to assess the efficiency of this silencing. MTT and apoptosis assays were conducted to detect cell viability and apoptosis, respectively. Compared with their parental cells, HCT-8/5-FU and Colo205/5-FU cells were 23.1 and 15.8 times more resistant to 5-FU, and 17.2 and 20.9 times more resistant oxaliplatin, respectively. The knockdown of MRP1 resulted in the attenuation of the MDR phenotype through the induction of apoptosis. The shMRP1-transfected Colo205/5-FU cells were injected subcutaneously into the right scapular region of $\mathrm{BALB} / \mathrm{c}$ nude mice and tumor size was measured for 15 days post-injection. This in vivo experiment demonstrated that MRP1 knockdown inhibited tumor growth. On the 9,12 and 15th day post-injection, tumor volume in the shMRP1-transfected Colo205/5-FU cell-injected group was significantly lower compared with that in the Colo205/5-FU cell-injected group (day 9, $2.1 \pm 0.8$ vs. $6.9 \pm 1.9 \mathrm{~mm}^{3}, \mathrm{P}=0.009$; day $12,3.1 \pm 1.4$ vs. $14.3 \pm 4.0 \mathrm{~mm}^{3}, \mathrm{P}=0.008$; day $15,4.8 \pm 2.7$ vs. $21.3 \pm 3.4 \mathrm{~mm}^{3}$; all $\left.\mathrm{P}<0.001\right)$. These results demonstrate that MRP1 serves a role in the MDR phenotype of CRC through inhibiting apoptosis and may serve as a potential therapeutic target for inhibition, which would increase the efficacy of other chemotherapeutic agents in the treatment of CRC.
\end{abstract}

Correspondence to: Professor Ming Zhong, Department of Gastrointestinal Surgery, Renji Hospital Affiliated to Shanghai Jiao Tong University School of Medicine, 160 Pujian Road, Pudong, Shanghai 200127, P.R. China

E-mail: surgeonzhongming@163.com

Key words: colorectal cancer, multidrug resistance, multidrug resistance associated protein 1, RNA interference, apoptosis

\section{Introduction}

Colorectal cancer (CRC) is the third most common type of cancer and has the third highest rate of cancer-associated mortality worldwide (1). In China, the incidence rate of CRC has steadily increased over the past 30 years, which has placed a substantial burden on individuals and society (2). Chemotherapy is used in the adjuvant and conversion therapy of patients with advanced CRC. Although significant advances in chemotherapy have been achieved, intrinsic or acquired chemoresistance, in particular multidrug resistance (MDR), is a major barrier to this development, resulting in the inefficient killing of cancer cells and subsequent patient relapse (3). MDR is defined as the simultaneous resistance to a range of structurally and mechanistically unrelated anticancer drugs. Therefore, it is important to better understand the biological mechanisms underlying MDR, in order to improve the efficacy of treatment for patients with CRC and other types of cancer.

The transporter hypothesis of drug resistance states that MDR is due to the upregulation of the ATP-binding cassette (ABC) family of membrane transporters, including multidrug resistance proteins (MRPs) and P-glycoprotein, which mediate the efflux of chemotherapeutic drugs from cancer cells (4). MDR associated protein 1 (MRP1) belongs to the MRP family and has been associated with MDR; however, its role in the MDR phenotype of CRC remains in dispute. Several studies identified no association between MRP1 expression and CRC-associated MDR (5-7). However, other studies identified high expression levels of MRP1 in CRC cells and produced results that indicated that there was a significant correlation between MRP1 expression and tumor stage/poor patient prognosis, in addition to an association with acquired chemoresistance, in CRC (8-11). The results of a study performed by Xing et al (10) suggest that MRP1 expression is not correlated with the functional changes of MRP1 transporters, which may partially explain the inconsistency in the results of the studies discussed above. Therefore, functional experiments are warranted to clarify the role of MRP1 in the MDR of CRC.

The role of MRP1 in the MDR phenotype of CRC was investigated in the present study and its potential as a therapeutic candidate was evaluated. The human MDR CRC cell lines HCT-8/5-fluorouracil (5-FU) and Colo205/5-FU were established from their parental cells. In vitro and in vivo experiments were subsequently conducted to investigate the effect of MRP1 knockdown on the response of MDR CRC cells to chemotherapy. 


\section{Materials and methods}

Cell lines and cell culture. Human CRC cell lines Colo205 and HCT- 8 were obtained from the Shanghai Institute of Biochemistry and Cell Biology (Chinese Academy of Sciences, Shanghai, China). The cells were cultured in Dulbecco's modified Eagle's medium (DMEM) supplemented with $10 \%$ fetal bovine serum (FBS) (both Hyclone; GE Healthcare Life Sciences, Logan, UT, USA) and incubated at $37^{\circ} \mathrm{C}$ with $5 \%$ $\mathrm{CO}_{2}$ in a humidified atmosphere.

Establishment of 5-FU resistant Colo205/5-FU and HCT-8/5-FU cell lines. The 5-FU resistant human CRC cell lines, Colo205/5-FU and HCT-8/5-FU, were established through the addition of increasing concentrations of 5-FU (0.01-2 $\mu \mathrm{g} / \mathrm{ml}$; Shanghai Lichen Biotechnology Co., Ltd., Shanghai, China) to the RPMI-1640 medium (Gibco; Thermo Fisher Scientific, Inc., Waltham, MA, USA) that the parental cells were cultured in. The concentration of 5-FU was increased between every 2 and 6 weeks, depending on the dosage required, for a total of 5 months. Cultures with $\geq 90 \%$ survival in the presence of $2 \mu \mathrm{g} / \mathrm{ml}$ of 5 -FU were maintained at $1 \mu \mathrm{g} / \mathrm{ml} 5-\mathrm{FU}$ and selected for subsequent experiments. The 5-FU resistant cells were snap-frozen in drug-free liquid nitrogen, and recovered and grown for 1 month in drug-free medium prior to collection at the asynchronous logarithmic phase of growth for subsequent experiments.

Knockdown of MRPI gene expression using RNAi. The sequence of the short hairpin (sh)RNA targeting MRP1 (Shanghai Genechem Co., Ltd., Shanghai, China) was sense, 5'-GCUGGUAGCCCUAGUGUGU-3' and anti-sense, 5'-ACA CACUAGGGCUACCAGC-3'. A non-specific control shRNA (sense, 5'-TTCTCCGAACGTGTCACGT-3' and anti-sense, 5'-ACGTGACACGTTCGGAGAA-3') was used as a negative control (Shanghai Genechem Co., Ltd.). Colo205/5-FU and HCT-8/5-FU cells were seeded at a density of $5 \times 10^{4}$ cells/well into 6-well plates for $24 \mathrm{~h}$ prior to transfection. Cells were transfected with anti-MRP1 shRNA (shMRP1; 2, 4 or $6 \mu \mathrm{g}$ per well) using Lipofectamine $2000^{\circledR}$ (Thermo Fisher Scientific, Inc.) according to the manufacturer's protocols.

Western blot analysis. A total of $48 \mathrm{~h}$ following shRNA transfection, soluble protein extracts were prepared through lysing cells in radioimmunoprecipitation assay (RIPA) buffer (Amyjet Scientific, Inc., Wuhan, China) in ice for $20 \mathrm{~min}$. Cells were lysed using three cycles of sonication on ice at $4-5 \mathrm{sec}$ per cycle (power, $60 \mathrm{~W}$ ), followed by centrifugation at $1200 \mathrm{x} g$ at $25^{\circ} \mathrm{C}$ for $10 \mathrm{~min}$. Protein quantification was performed using a BCA Protein Assay kit (Bio-Rad Laboratories, Inc., Hercules, CA, USA). Proteins were denatured at $100^{\circ} \mathrm{C}$ for $5 \mathrm{~min}$. Equal amounts of total protein extract $(50 \mu \mathrm{g})$ were loaded and separated via SDS-PAGE on a $12 \%$ gel, and transferred onto polyvinylidene difluoride membranes (Thermo Fisher Scientific, Inc.). The membranes were blocked with skimmed milk (5\%) dissolved in Tris-buffered saline containing $0.05 \%$ Tween-20 for $2 \mathrm{~h}$ at room temperature (RT). Membranes were subsequently incubated overnight at $4^{\circ} \mathrm{C}$ with a primary anti-MRP1 antibody (cat no. MAB2388; 1:1,000; Abnova, Taipei, Taiwan), followed by incubation with a horseradish peroxidase (HRP)-conjugated goat anti-mouse secondary antibody (cat no. BA1050-1; 1:8,000; Wuhan Boster Biological Technology, Ltd., Wuhan, China) for $1 \mathrm{~h}$ at RT. Protein bands were visualized using the ECL Plus ${ }^{\mathrm{TM}}$ Western Blotting Detection kit (GE Healthcare Life Sciences, Chalfont, UK) and subsequent exposure was performed using a luminescent image analyzer (LAS-1000; Fujifilm, Tokyo, Japan).

Parallel membranes, the same as that blotted above, were incubated with a rabbit anti- $\beta$-actin monoclonal antibody (cat no. A 2668; 1:10,000; Sigma-Aldrich; Merck Millipore, Darmstadt, Germany) at $4^{\circ} \mathrm{C}$ overnight, followed by a HRP-conjugated goat anti-rabbit secondary antibody (cat no. ab205718; 1:20,000; Abcam, Cambridge, UK) for $1 \mathrm{~h}$ at RT. The relative level of MRP1 protein was normalized to $\beta$-actin in the untransfected cells using ImageJ software (version 1.37; National Institutes of Health, Bethesda, MD, USA).

Cell viability assay. The cytotoxicity of 5-FU $(1 \mu \mathrm{g} / \mathrm{ml})$ and oxaliplatin $(l-\mathrm{OHP})(2 \mu \mathrm{g} / \mathrm{ml})$ (both Shanghai Lichen Biotechnology Co., Ltd.) were determined using the MTT assay-based Cell Growth Determination kit (Sigma-Aldrich; Merck Millipore), as described previously (12). Briefly, cells were cultured in a flat 96-well plate at a density of 1,000-10,000 cells/well until $\sim 80 \%$ confluence was reached. Cells were harvested using trypsin, resuspended in DMEM containing 10\% FBS and centrifuged at $1,200 \mathrm{x} g$ at $25^{\circ} \mathrm{C}$ for $10 \mathrm{~min}, 12,24,36,48$ and $60 \mathrm{~h}$ following drug exposure. Absorbance was measured at $492 \mathrm{~nm}$ using a microplate reader. The half maximal inhibitory concentration $\left(\mathrm{IC}_{50}\right)$, defined as the drug concentration required to reduce cell survival to $50 \%$ compared to untreated cells, as determined by the relative absorbance of MTT, was assessed through probit regression analysis. The resistance index (\%) for each drug was calculated as follows: $\left(\mathrm{IC}_{50}\right.$ of treated cells / $\mathrm{IC}_{50}$ of untreated cells) x100.

Apoptosis assay. An apoptosis assay was performed to determine the effect of MRP1 knockdown on the apoptosis of CRC cells in response to 5-FU. Apoptosis was detected using the Annexin V-FITC Apoptosis Detection kit (eBioscience, Inc., San Diego, CA, USA). A total of $48 \mathrm{~h}$ following shRNA transfection, Colo205/5-FU, HCT-8/5-FU cells and controls (Colo205/5-FU and HCT-8/5-FU cells transfected with scramble shRNA) were exposed to $0.2 \mu \mathrm{g} / \mathrm{ml} 5$-FU for $48 \mathrm{~h}$ and subsequently harvested through trypsinization, washing twice in ice-cold PBS and centrifugation at $1200 \mathrm{x} g$ at $4^{\circ} \mathrm{C}$ for $5 \mathrm{~min}$. Cells were then washed twice with ice-cold PBS and resuspended in binding buffer from the Annexin V-FITC Apoptosis Detection kit. Annexin V-FITC protein $(0.5 \mu \mathrm{g} / \mathrm{ml})$ was added to the cell suspension, which was incubated for $10 \mathrm{~min}$ at RT. The cells were washed in PBS, resuspended in binding buffer and incubated with propidium iodide $(0.6 \mu \mathrm{g} / \mathrm{ml})$ for $15 \mathrm{~min}$ in the dark at RT. The stained cells were analyzed using the BD FACSCalibur $^{\mathrm{TM}}$ flow cytometer (BD Biosciences, San Jose, CA, USA). BD Accuri ${ }^{\mathrm{TM}}$ C6 software (version 1.0.264.21; BD Biosciences) was used for the quantification of apoptosis.

Animal experiments. All animal procedures were conducted in accordance with The Declaration of Helsinki and the Guide for the Care and Use of Laboratory Animals (National Institutes of Health). In addition, all procedures 
Table I. Establishment and validation of the multidrug resistance phenotype of colorectal cancer cell lines.

\begin{tabular}{lccc}
\hline Cell line & $\mathrm{IC}_{50} 5$-FU $[$ mean $\pm \mathrm{SD}(\mathrm{mg} / \mathrm{l})]$ & $\mathrm{RI}$ & $\mathrm{IC}_{50} l$-OHP $[\mathrm{mean} \pm \mathrm{SD}(\mathrm{mg} / \mathrm{l})]$ \\
\hline HCT-8 & $0.0106 \pm 0.004$ & 15.8 & $0.0063 \pm 0.004$ \\
HCT-8/5-FU & $0.2657 \pm 0.003$ & & $0.1134 \pm 0.005$ \\
Colo205 & $0.0066 \pm 0.008$ & 23.1 & $0.0097 \pm 0.004$ \\
Colo205/5-FU & $0.1181 \pm 0.002$ & & $0.1101 \pm 0.062$
\end{tabular}

n=3/group. HCT-8/5-FU and Colo205/5-FU cells were more resistant to 5-FU compared with their parental cells. HCT-8/5-FU and Colo205/5-FU cells also exhibited cross-resistance to $l$-OHP. 5-FU, 5-fluorouracil; $l$-OHP, oxaliplatin; IC $_{50}$, half maximal inhibitory concentration; RI, resistance index.

involving animals were reviewed and approved by the Ethics Committee of Renji Hospital (Shanghai, China). A total of 20 male BALB/c nude mice (5 weeks old; 18-22 g; Shanghai Laboratory Animal Centre, Shanghai, China) were used in the present study. Mice were kept under a 12-h-light/dark cycle at $22 \pm 2^{\circ} \mathrm{C}$ and $60 \pm 5 \%$ relative humidity. Food and water were supplied ad libitum. A total of $4 \times 10^{6}$ Colo205/5-FU cells were resuspended in $100 \mu \mathrm{l}$ PBS and injected subcutaneously into the right scapular region of each mouse $24 \mathrm{~h}$ following shRNA transfection. The length and width of the resulting tumor mass at the inoculation site was measured using a caliper every 3 days for 15 days post injection, in order to assess tumor growth.

Statistical analysis. Each experiment was performed $\geq 3$ times. Results are presented as the mean \pm standard deviation. One-way analysis of variance was used, followed by Dunnett's test (two-tailed). Probit regression analysis was used to calculate $\mathrm{IC}_{50}$ in cell viability assay. $\mathrm{P}<0.05$ was considered to indicate a statistically significant difference. All statistical analyses were carried out using SPSS software (version 15.0; SPSS, Inc., Chicago, IL, USA).

\section{Results}

Establishment and validation of the MDR phenotype of the Colo205/5-FU and HCT-8/5-FU cell lines. To ascertain whether HCT-8/5-FU and Colo205/5-FU cells exhibited stable resistance to $5-\mathrm{FU}$, the $\mathrm{IC}_{50}$ values for 5-FU in the MDR CRC cells and their parental cells were examined. HCT-8/5-FU and Colo205/5-FU cells were 15.8 and 23.1 times more resistant to 5-FU, respectively, compared with their parental cells (Table I). Furthermore, the cell lines exhibited cross-resistance to $l$-OHP (Table I).

RNAi decreases MRPI protein expression in MDR cells. The efficacy of shMRP1 silencing of MRP1 was assessed using western blotting. Prior to MDR induction in Colo205 and HCT-8 cells, MRP1 protein expression was detectable (Fig. 1). Following MDR induction, MRP1 protein expression increased notably in the cell lines (Fig. 1C). Conversely, the expression levels of MRP1 protein in the MDR CRC cells were reduced following $2 \mu \mathrm{g}$ shRNA transfection compared with the untreated MDR cells (HCT-8/5-FU shRNA group, $\mathrm{P}=0.133$; Colo205/5-FU shRNA group, $\mathrm{P}=0.161$; Fig. 1C).
Therefore, $2 \mu \mathrm{g}$ of shMRP1 was used for the transfection of Colo205 and HCT-8 cells in subsequent studies.

Silencing MRPI attenuates the response of MDR CRC cells to 5-FU and l-OHP. Silencing of MRP1 notably increased chemosensitivity to 5 -FU and $l$-OHP in the MDR CRC cell sublines compared with the untransfected cells (Table IIA). To achieve $50 \%$ inhibition of the shMRP1-transfected Colo205/5-FU cells, $\geq 10 \%$ of the amount of 5-FU was required compared with the untransfected Colo205/5-FU controls. Drug resistance in the MDR CRC cell sublines was restored to the levels similar to those observed in the parental cell lines when shMRP1 was transfected into the MDR cells (Table IIB).

Silencing of MRP1 enhances apoptosis in HCT-8/5-FU and Colo205/5-FU cells. An apoptosis assay was performed to assess the effect of MRP1 knockdown on the apoptosis of MDR GC cells (Fig. 2). The percentage of apoptotic shMRP1-transfected Colo205/5-FU cells was significantly higher compared with cells in the untransfected control group (8.11 vs. $0.11 \%$; $\mathrm{P}<0.001$; Fig. $2 \mathrm{~A}$ and C). Similarly, the apoptotic rate of shMRP1-transfected HCT-8/5-FU cells increased significantly compared with HCT-8/5-FU cells (8.50 vs. $0.14 \%$; $\mathrm{P}=0.001$; Fig. 2B and C).

shRNA-induced MRPI knockdown has an anticancer effect in $B A L B / c$ nude mice. During the first 4 days post-injection of untransfected or shRNA-transfected Colo205/5-FU cells, no notable difference in tumor size was observed between the two groups (Fig. 3). Between days 4 and 14, tumor growth increased markedly in the Colo205/5-FU cell-injected group compared with the ShMRP1-transfected Colo205/5-FU cell-injected group (Fig. 3B). On the 9, 12 and 15th day post-injection, tumor volume in the shMRP1-transfected Colo205/5-FU cell-injected group was significantly lower compared with the Colo205/5-FU cell-injected group (day 9, 2.1 \pm 0.8 vs. $6.9 \pm 1.9 \mathrm{~mm}^{3}, \mathrm{P}=0.009$; day $12,3.1 \pm 1.4$ vs. $14.3 \pm 4.0 \mathrm{~mm}^{3}$, $\mathrm{P}=0.008$; day $15,4.8 \pm 2.7$ vs. $21.3 \pm 3.4 \mathrm{~mm}^{3}, \mathrm{P}<0.001$; Fig. $3 \mathrm{~B}$ ).

\section{Discussion}

For the majority of patients with CRC there is a high risk of tumor relapse and metastasis, even following radical resection of the tumor (13). Therefore, chemotherapeutic agents (fluoropyrimidines, platinum, anthracyclines and others) serve 
Table II. Restoration of chemosensitivity following MRP1 knockdown.

A, RNAi knockdown of MRP1 resensitized 5-FU-resistant CRC cells to 5-FU and $l$-OHP.

\begin{tabular}{lccc}
\hline Cell line & $\mathrm{IC}_{50} 5$-FU $[$ mean $\pm \mathrm{SD}(\mathrm{mg} / \mathrm{l})]$ & $\mathrm{RI}$ & $\mathrm{IC}_{50} l$-OHP $[\mathrm{mean} \pm \mathrm{SD}(\mathrm{mg} / \mathrm{l})]$ \\
\hline HCT-8/5-FU & $0.2657 \pm 0.003$ & 0.23 & $0.1134 \pm 0.005$ \\
HCT-8/5-FU/ShMRP1 & $0.0608 \pm 0.033$ & & $0.0116 \pm 0.002$ \\
Colo205/5-FU & $0.1181 \pm 0.002$ & 0.09 & $0.1101 \pm 0.062$ \\
Colo205/5-FU/ShMRP1 & $0.0108 \pm 0.006$ & & $0.0169 \pm 0.022$ \\
\hline
\end{tabular}

B, RNAi knockdown of MRP1 reversed 5-FU sensitivity

\begin{tabular}{lccc}
\hline Cell line & $\mathrm{IC}_{50} 5$-FU $[$ mean $\pm \mathrm{SD}(\mathrm{mg} / \mathrm{l})]$ & $\mathrm{RI}$ & $\mathrm{IC}_{50} l$-OHP $[\mathrm{mean} \pm \mathrm{SD}(\mathrm{mg} / \mathrm{l})]$ \\
\hline HCT-8 & $0.0106 \pm 0.004$ & 5.73 & $0.0066 \pm 0.008$ \\
HCT-8/5-FU/ShMRP1 & $0.0608 \pm 0.033$ & & $0.0108 \pm 0.006$ \\
Colo205 & $0.0063 \pm 0.004$ & 1.84 & $0.0097 \pm 0.004$ \\
Colo205/5-FU/ShMRP1 & $0.0116 \pm 0.002$ & & $0.0169 \pm 0.022$
\end{tabular}

$\mathrm{n}=3$ /group. MRP1, multidrug resistance associated protein 1; RNAi, RNA interference; 5 -FU, 5-fluorouracil; $l$-OHP, oxaliplatin; $\mathrm{IC}_{50}$, half maximal inhibitory concentration; RI, resistance index.
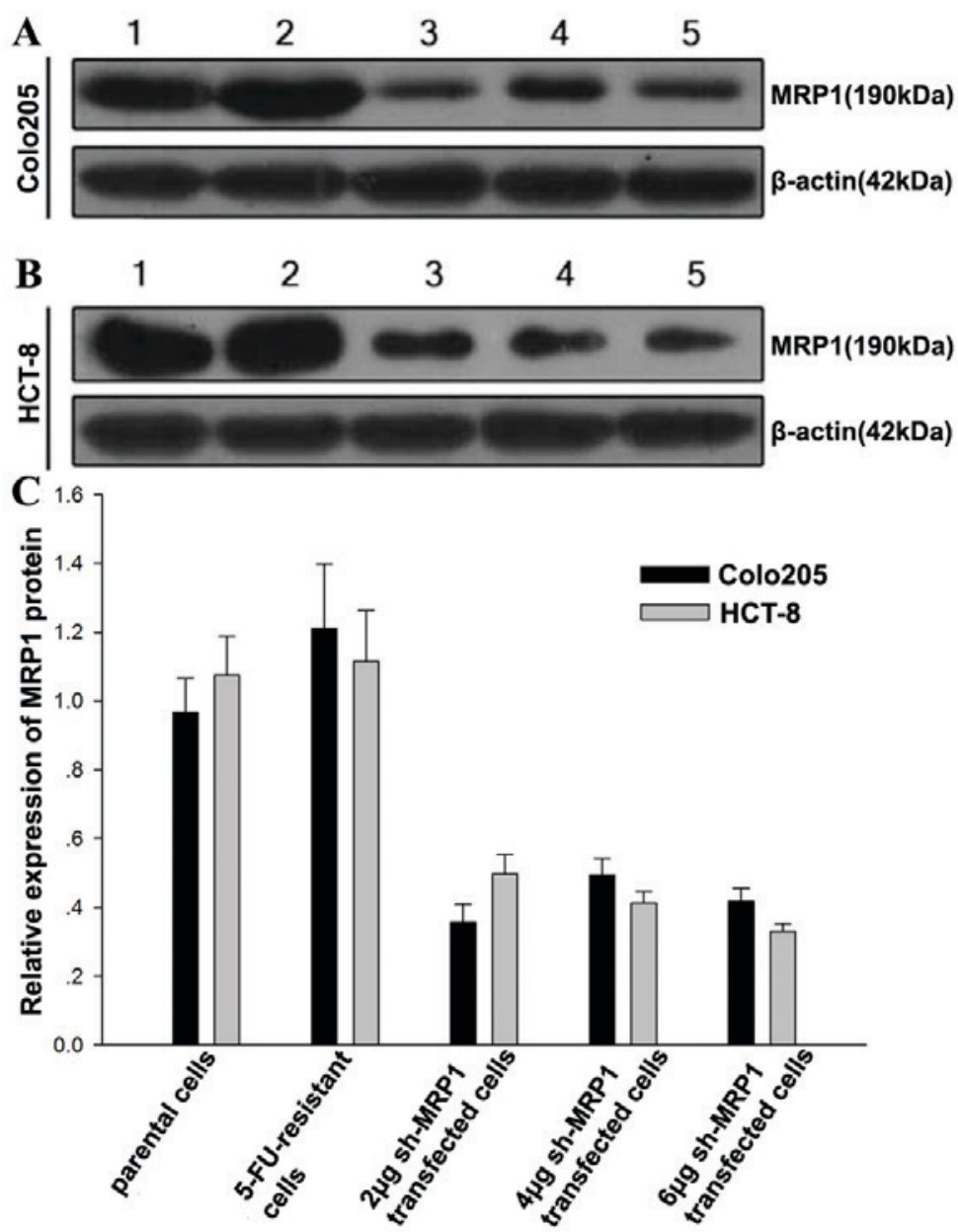

Figure 1.MRP1 protein expression in multidrug resistance colorectal cancer cells. Representative western blot of MRP1 protein expression in (A) Colo205/5-FU and (B) HCT-8/5-FU cells for five independent samples. Lanes: 1, parental cells; 2, 5-FU-resistant cells; 3, $2 \mu \mathrm{g}$ shRNA-transfected 5-FU resistant cells; 4, $4 \mu \mathrm{g}$ shRNA-transfected 5-FU resistant cells; 5, $6 \mu \mathrm{g}$ shRNA-transfected 5-FU resistant cells. The expression of MRP1 markedly diminished following treatment with shMRP1. (C) Results of relative MRP1 protein expression in Colo205/5-FU and in HCT-8/5-FU cells. $\mathrm{n}=3 /$ group. Results are presented as the mean \pm standard error. MRP1, multidrug resistance associated protein 1; shRNA, short hairpin RNA; shMRP1, shRNA targeting MRP1; 5-FU, 5-fluorouracil. 
A

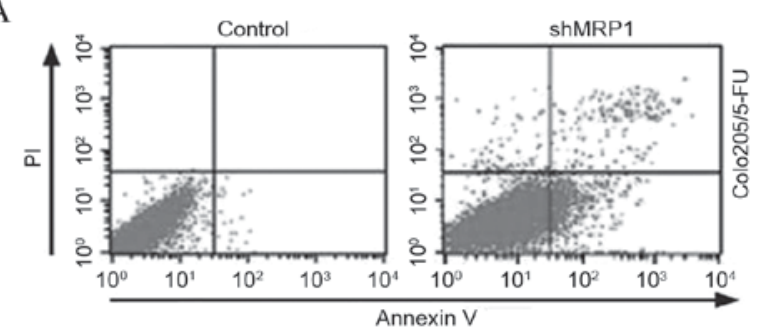

B
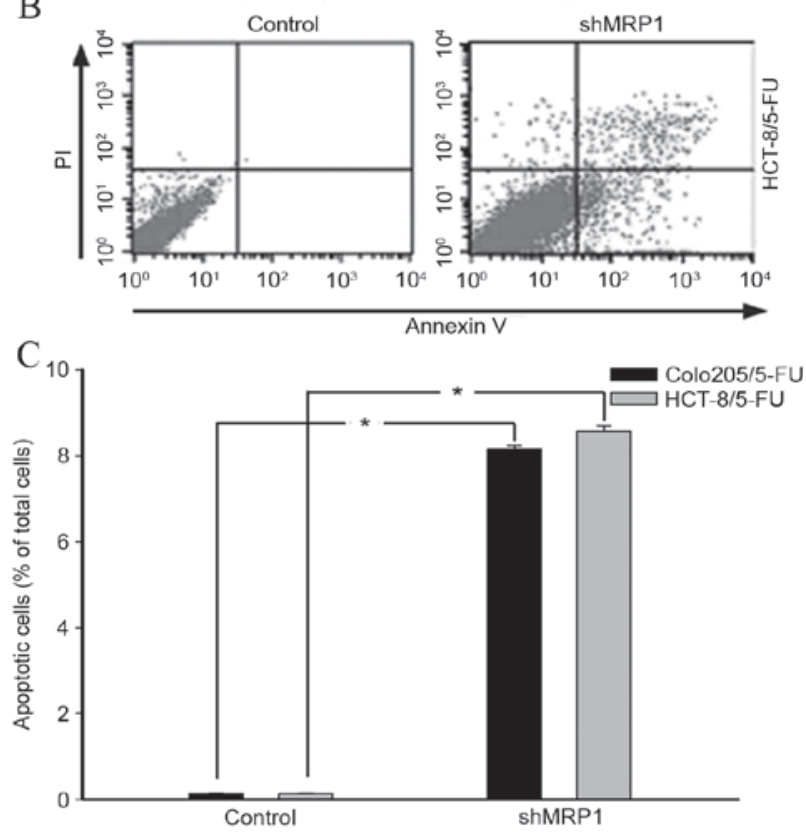

Figure 2. Apoptosis of multidrug resistance colorectal cancer cells following shMRP1 transfection. The percentage of cell apoptosis following shMRP1 transfection for (A) Colo205/5-FU cells and (B) HCT-8/5-FU cells (C) Apoptosis was significantly increased in hMRP1-transfected 5-FU resistant cells compared with their untransfected counterparts. n=3/group ${ }^{*} \mathrm{P}<0.001$. shMRP1, short hairpin RNA targeting multidrug resistance associated protein 1; PI, propidium iodide; 5-FU, 5-fluorouracil.

an essential role in the treatment of such cases. Despite being potent anticancer agents, these drugs may become ineffective in the treatment of various types of cancer due to the development of acquired resistance. Hence, overcoming MDR is essential to enhance tumor chemosensitivity and improve the prognosis of patients with CRC. The molecular mechanisms underlying MDR remain unclear. MRP family proteins are involved in MDR in various diseases, including cancer (14). Recently, the role of MRPs in CRC MDR been investigated in more detail, revealing that $\mathrm{ABC}$-transporters are upregulated in a subpopulation of MDR CRC stem cells (15). Further research into the role of MRPs in the MDR phenotype of CRC is warranted.

There are conflicting reports regarding the association between the expression of MRP1, a member of the MRP family, and MDR in CRC. Nakamura et al (5) and Nishioka et al (6) identified no significant association between MRP1 mRNA levels or its genotypes and CRC chemosensitivity. In addition, Lee et al (7) identified that the level of MRP1 protein in $\mathrm{CRC}$ tissue was not associated with the $\mathrm{IC}_{50}$ of various anticancer drugs (5-FU, irinotecan and $l$-OHP), as assessed using histoculture drug response assays. Conversely, MRP1 overexpression was observed in patients with metastatic CRC

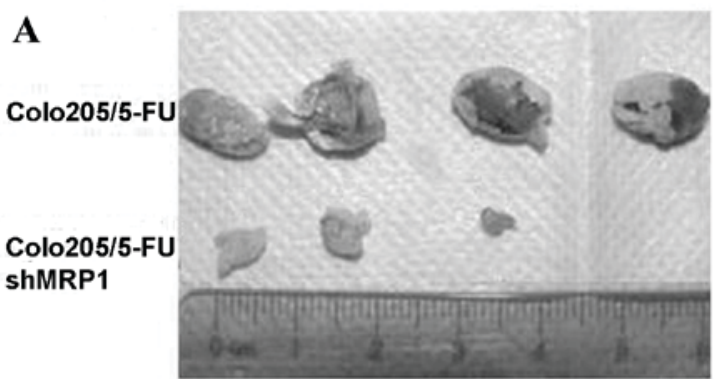

B

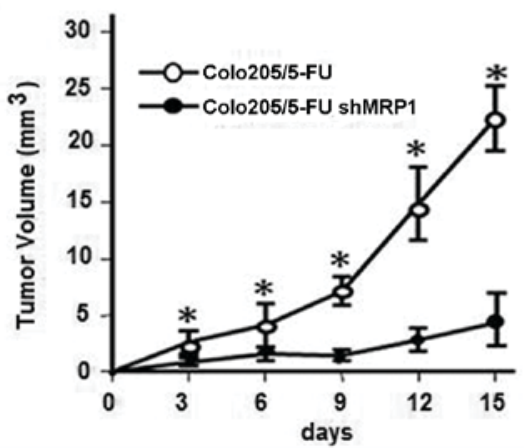

Figure 3. Short hairpin RNA-induced MRP1 knockdown inhibits colorectal cancer tumor growth in BALB/c nude mice. (A) Tumor volume was measured using a caliper and the measurement of tumor volume in the 2 groups was displayed on the 15th day following shMRP1-transfected Colo205/5-FU cell injection. (B) On the 9, 12 and 15th day tumor volume in the shMRP1-transfected Colo205/5-FU cell-injected group was significantly lower compared with that in the Colo205/5-FU cell-injected group. $n=4$ /group. ${ }^{*} \mathrm{P}<0.001$ vs. untransfected cell-injected mice. shMRP1, short hairpin RNA targeting multidrug resistance associated protein 1; 5-FU, 5-fluorouracil.

who had undergone chemotherapy, demonstrating the influence of chemotherapy on the function of MRP1 transporters in CRC cells (8). Recently, Ji et al (9) reported elevated MRP1 expression in CRC tissues, and identified a significant correlation between MRP1 expression and Dukes' stage, in addition to poor patient prognosis. Micsik et al (11) suggested that MDR activity may be associated with acquired chemoresistance in CRC. Furthermore, another study suggested that MRP1 inhibition was involved in the fingolimod-induced chemosensitization of HCT-8/5-FU cells (10). Notably, the present study demonstrated that MRP1 participates in the FTY720-induced chemosensitization effect in HCT-8 and HCT-8/5-Fu cell lines. This suggests that MRP1 gene expression in CRC tissue was not significantly correlated with the functional changes of MRP1 transporters, which may partially explain the discrepancy between the results of previous studies (10). Functional experiments focusing on the alteration of MRP1 pump transporter activity in MDR CRC are required.

The present study investigated the effect of MRP1 in the development of MDR in CRC through in vitro and in vivo functional experiments. Tolerance to 5-FU in HCT-8/5-FU and Colo205/5-FU cells was associated with an upregulation of MRP1 protein expression. HCT-8/5-FU and Colo205/5-FU cells were 15.8 and 23.1 times more resistant to 5-FU, respectively, compared with their untransfected counterparts. This is consistent with the results of a previous study, which indicated that MRP1 overexpression is associated with resistance to 5-FU (16). In addition, HCT-8/5-FU and Colo205/5-FU cells exhibited cross-resistance to $l$-OHP that was 17.2 and 
20.9 times stronger, respectively, compared with that of their untransfected counterparts, demonstrating the induction of a MDR phenotype. Following MRP1 knockdown, the reduction of MRP1 protein expression was associated with RI values that reached levels comparable with that of the parental cells, illustrating that the MDR phenotype was reversible. Furthermore, in vivo studies in nude mice revealed that MRP1 knockdown increased the chemosensitivity of MDR CRC cells to 5-FU. MRP1 knockdown inhibited tumor growth in Colo205/5-FU cell-injected mice between days 4 and 15 post-injection.

The results of the present study revealed that MRP1 knockdown sensitized MDR CRC cells to 5 -FU and $l$-OHP. Currently, FOLFOX (folinic acid, 5-fluorouracil and $l$-OHP) and XELOX (capecitabine and $l$-OHP) are the first-line chemotherapy regimens for CRC (16). These regimens use 5 -FU, or its analogue capecitabine, and $l$-OHP as their active components. The present study demonstrated the potential of MRP1 as a therapeutic target in the treatment of CRC with a MDR phenotype and will prompt efforts to develop agents that are able to repress MRP1-mediated drug transport. Currently, an antibody selective for MRP1 has been isolated; however, its specificity and efficiency as a chemosensitizer in the treatment of CRC has not yet been reported (17). Another potential treatment option is the post-transcriptional interference of MRP1 expression, as suggested by the results of the present study. Advances in the molecular biology techniques, including RNAi-based treatment and nanotechnology-mediated delivery of treatments, are required to develop novel treatments for MDR CRC that have a higher efficacy and lower toxicity compared with current chemotherapy treatments $(18,19)$.

The current study revealed that MRP1 knockdown enhanced the chemotherapeutic response of MDR CRC cells via increasing apoptosis. In various types of cancer, an association between the deregulation of apoptosis and a MDR phenotype has been established. MRP1 belongs to the ABC transporter family of proteins, which contribute to cellular detoxification through the efflux of various molecules from the cytoplasm, thus preventing the accumulation of cytotoxic compounds in cells (20). It was presumed that MRP1 activation enhances chemoresistance through producing an anti-apoptotic effect via reducing intracellular drug concentrations to below therapeutic levels. This is in agreement with the finding that $l$-OHP and its bioactive moieties are substrates of MRP1; suggesting that inhibition of MRP1 may result in increased efficacy of $l$-OHP (16).

In conclusion, the present study demonstrated the effect of MRP1 on the MDR phenotype of CRC using in vitro and in vivo techniques. The results of the current study indicated that MRP1 increases CRC cell resistance to apoptosis. Thus, inhibition of MRP1 is an attractive strategy to overcome MDR in CRC. Further studies are warranted to investigate the prognostic significance of MRP1, in addition to the potential of MRP1 inhibition as an approach to chemosensitivity modulation, which is expected to contribute to the personalized treatment of patients with CRC in the future.

\section{Acknowledgements}

The present study was supported by the National Natural Science Foundation of China (grant no. 81201806) and the Natural Science Foundation of Shanghai (grant no. 12ZR1442000).

\section{References}

1. Siegel RL, Miller KD and Jemal A: Cancer statistics, 2015. CA Cancer J Clin 65: 5-29, 2015.

2. Li L and Ma BB: Colorectal cancer in Chinese patients: Current and emerging treatment options. Onco Targets Ther 7: 1817-1828, 2014.

3. Gustavsson B, Carlsson G, Machover D, Petrelli N, Roth A, Schmoll HJ, Tveit KM and Gibson F: A review of the evolution of systemic chemotherapy in the management of colorectal cancer. Clin Colorectal Cancer 14: 1-10, 2015.

4. Kathawala RJ, Gupta P, Ashby CR Jr and Chen ZS: The modulation of $\mathrm{ABC}$ transporter-mediated multidrug resistance in cancer: A review of the past decade. Drug Resist Updat 18: 1-17, 2015.

5. Nakahara T, Sakaeda T, Nakamura T, Tamura T, Nishioka C, Aoyama N, Okamura N, Shirakawa T, Gotoh A, Kamigaki T, et al: Chemosensitivity assessed by collagen gel droplet embedded culture drug sensitivity test, and MDR1, MRP1, and MRP2 mRNA expression in human colorectal adenocarcinomas. Pharm Res 21: 406-412, 2004.

6. Nishioka C, Sakaeda T, Nakamura T, Moriya Y, Okamura N, Tamura T, Nakahara T, Aoyama N, Kamigaki T, Ohno M, et al: MDR1, MRP1 and MRP2 genotypes and in vitro chemosensitivity in Japanese patients with colorectal adenocarcinomas. Kobe J Med Sci 50: 181-188, 2004.

7. Lee JH, Um JW, Lee JH, Kim SH, Lee ES and Kim YS: Can immunohistochemistry of multidrug-resistant proteins replace the histoculture drug response assay in colorectal adenocarcinomas? Hepatogastroenterology 59: 1075-1078, 2012.

8. Nanashima A, Yamaguchi H, Matsuo S, Sumida Y, Tsuji T, Sawai T, Yasutake T, Nakagoe T and Ayabe H: Expression of multidrug resistance protein in metastatic colorectal carcinomas. J Gastroenterol 34: 582-588, 1999.

9. Ji L, Wei Y, Jiang T and Wang S: Correlation of Nrf2, NQO1, MRP1, cmyc and p53 in colorectal cancer and their relationships to clinicopathologic features and survival. Int J Clin Exp Pathol 7: 1124-1131, 2014.

10. Xing Y, Wang ZH, Ma DH and Han Y: FTY720 enhances chemosensitivity of colon cancer cells to doxorubicin and etoposide via the modulation of P-glycoprotein and multidrug resistance protein 1. J Dig Dis 15: 246-259, 2014.

11. Micsik T, Lorincz A, Mersich T, Baranyai Z, Besznyák I Jr, Dede K, Zaránd A, Jakab F, Szöllösi LK, Kéri G, et al: Decreased functional activity of multidrug resistance protein in primary colorectal cancer. Diagn Pathol 10: 26, 2015.

12. Zhang J, Wang X, Liu T, Liu S and Jing X: Antitumor activity of electrospun polylactide nanofibers loaded with 5-fluorouracil and oxaliplatin against colorectal cancer. Drug Deliv 23: 794-800, 2016.

13. Van Cutsem E, Cervantes A, Nordlinger B and Arnold D; ESMO Guidelines Working Group: Metastatic colorectal cancer: ESMO clinical practice guidelines for diagnosis, treatment and follow-up. Ann Oncol 25 (Suppl 3): iii1-iii9, 2014.

14. Yu Z, Zhang C, Wang H, Xing J, Gong H, YuE, Zhang W, Zhang X, Cao $\mathrm{G}$ and Fu C: Multidrug resistance-associated protein 3 confers resistance to chemoradiotherapy for rectal cancer by regulating reactive oxygen species and caspase-3-dependent apoptotic pathway. Cancer Lett 353: 182-193, 2014.

15. Cui H, Zhang AJ, Chen M and Liu JJ: ABC transporter inhibitors in reversing multidrug resistance to chemotherapy. Curr Drug Targets 16: 1356-1371, 2015.

16. Theile D, Grebhardt S, Haefeli WE and Weiss J: Involvement of drug transporters in the synergistic action of FOLFOX combination chemotherapy. Biochem Pharmacol 78: 1366-1373, 2009.

17. Binyamin L, Assaraf YG, Haus-Cohen M, Stark M and Reiter Y: Targeting an extracellular epitope of the human multidrug resistance protein 1 (MRP1) in malignant cells with a novel recombinant single chain Fv antibody. Int J Cancer 110: 882-890, 2004.

18. Peer D and Howard KA: RNA interference-based therapeutics and diagnostics. Drug Deliv Transl Res 4: 1-2, 2014.

19. Gao Y, Shen JK, Milane L, Hornicek FJ, Amiji MM and Duan Z: Targeted cancer therapy; nanotechnology approaches for overcoming drug resistance. Curr Med Chem 22: 1335-1347, 2015.

20. Quan E, Wang H, Dong D, Zhang X and Wu B: Characterization of chrysin glucuronidation in UGT1A1-overexpressing HeLa cells: Elucidating the transporters responsible for efflux of glucuronide. Drug Metab Dispos 43: 433-443, 2015. 